A CONTRIBUTION TO THE BIO-CHEMISTRY OF HAEMOLYSIS :-

(a) CHANGES IN SOLUBILITY OF THE LIPOIDS IN PRESENCE OF ONE ANOTHER, AND OF CERTAIN UNSATURATED ORGANIC SUBSTANCES.

(b) THE BALANCING ACTION OF CERTAIN PAIRS OF HAEMOLYSERS IN PREVENTING HAEMOLYSIS.

(c) THE PROTECTIVE ACTION OF SERUM PROTEINS AGAINST HAEMOLYSERS.

(d) THE EFFECTS OF OXYDISING AND REDUCING AGENTS UPON HAEMOLYSIS.

By BENJAMIN MOORE, M.A., D.Sc. (R.t.I.), Johnston Professor of Bio-chemistry, University of Liverpool; FREDERICK P. WILSON, M.D. (Liverpool), AND LANCELOT HUTCHINSON, M.D. (Liverpool).

From the Department of Bio-chemistry, University of Liverpool (Received July 22nd, 1909)

The subject of haemolysis, and the relationship of lipoid substances to this process of laking of the blood corpuscles, is one which is at the present time exciting very general attention from physical chemists, biological chemists, and clinicians alike because of its important relationships to the chemistry of colloidal solutions on the one hand, and of its valuable applications to the diagnosis of disease on the other.

In earlier papers more directly concerned with the subject of the digestion and absorption of fats, it was shown in 1897 by Moore and Rockwood ${ }^{1}$ and in 1901 by Moore and Parker ${ }^{2}$ that the salts of the bile, and the products of fatty cleavage of a lipoidal nature, possessed when in common solution some kind of an affinity, apparently of a physicochemical nature, which had the effect of increasing the solubilities of the fatty acids and soaps.

This remarkable change in solubilities was shown by Moore and Parker to extend to other lipoids, such as lecithin. It was further demonstrated that the unsaturated oleic acid and its sodium soap had a

1. Proc. Roy. Soc., Vol. LX, p. 438, 1897; Journ. of Physiology, Vol. XXI, p. 58, 1897.

2. Proc. Roy. Soc., Vol. LXVIII, p. 64, 1901. 
most peculiar effect in increasing many times the solubilities of the fully saturated palmitic and stearic acids and their sodium soaps. These when in a state of purity were found to have practically $\dot{a}$ zero solubility in either water or bile salt solution.

Moore and Parker showed in the case of lecithin, which they prepared from egg yolk, that the lecithin, when it was added to a solution of bile salts or to bile at body temperature, did not form an emulsion or fine suspension as in the case of treatment with water, but gave instead a water clear solution. This solution was then more effective than the original in dissolving other lipoids.

They also found, probably on account of this mutual effect upon solubilities of the different lipoids, that bile as a whole was a much more effective solvent than a considerably stronger solution of the separated and re-dissolved bile salts.

These earlier results on mutual solubility appear to us to possess a bearing, which will be pointed out later, upon the process of haemolysis of the lecithin-containing corpuscles by other lipoids, such as sodium oleate, the bile salts, and saponin-like bodies, and for this reason we quote here certain of the figures given by Moore and Parker which definitely show the mutual effects.

The solubility of the fatty acids and soaps was found to be as follows :-

'Oleic Acid : solubility in distilled water less than 0.1 per cent.; solubility in 5 per cent. bile salt solution, about 0.5 per cent. ; solubility in 5 per cent. bile salts plus one per cent. lecithin, 4.0 per cent.'

'Palmitic Acid : in distilled water less than $0 \cdot 1$ per cent.; in 5 per cent. bile salts, about 0.1 per cent. ; in 5 per cent. bile salts plus 1 per cent. lecithin, 0.6 per cent.'

'Stearic Acid : in distilled water less than 0.1 per cent.; in 5 per cent. bile salts less than 0.1 per cent. ; in 5 per cent. bile salts plus 1 per cent. lecithin, 0.2 per cent.'

'Sodium Oleate : in distilled water, $5 \cdot 0$ per cent. ; in 5 per cent. bile salts, $7 \cdot 6$ per cent. ; in 5 per cent. bile salts plus 1 per cent. lecithin, $11 \cdot 6$ per cent.

'Sodium Palmitate : in distilled water, 0.2 per cent. ; in 5 per cent. bile salts, 1.0 per cent. ; in 5 per cent. bile salts plus 1 per cent. lecithin, 2.4 per cent.'

$\therefore$ Sodium Stearate: in distilled water, 0.1 per cent.; in 5 per cent. bile salts, 0.2 per cent. ; in 5 per cent. bile salts plus 1 per cent. lecithin, 0.7 per cent.'

'Lecithin. "Pure" lecithin is practically insoluble in water, the addition of as little as 0.1 per cent. causes an opalescence and further additions give rise, as is well known, to a kind of emulsion. But when lecithin is added to a 5 per cent. solution of bile salts, or to bile, the appearances observed are quite different.'

'The lecithin dissolves to a clear brown-coloured solution and the amount taken up is surprising; thus a 5 pær sent. solution of bile salts takes up no less than 7 per cent. of lecithin 
at a temperature of $37^{\circ} \mathrm{C}$. On cooling, part of the lecithin is thrown out of solution as a finely suspended precipitate or emulsion which glistens with a silky lustre when the test-tube containing it is shaken so as to set the fluid in motion. At ordinary room temperatures of $15^{\circ}$ to $20^{\circ} \mathrm{C}$. a considerable amount of lecithin, 4 to 5 per cent., is, however, still retained in solution.'

'The power of lecithin in increasing the solubilities of the fatty acids and soaps, explains in great part why lower solubilities are obtained in experimenting with pure bile salt solutions, than with bile. The lecithin naturally occurring in bile thus increases the solvent power of that fluid in the intestine for fatty acids and soaps.'

We have quoted at length these earlier experiments upon the mutual effects of different lipoids in common solution upon one another, because they appear to us to have some bearing upon haemolytic phenomena. For example, sodium oleate or sodium linoleate have a strong laking effect upon the red blood corpuscles. Now the red blood corpuscles contain lecithin, but the above experiments show that the presence of lecithin in solution increases the solubility of oleates. In haemolysis of this type it is hence obvious that the converse result is being obtained and that the oleates or linoleates are laking the corpuscles, because lecithin is more soluble in presence of the oleates or linoleates.

We shall also see that the bile salts and the members of the saponindigitalin group of glucosides are all unsaturated compounds like the oleates and linoleates, and that they increase by their presence lipoid solubilities, and hence are powerful laking agents.

These results upon solubility were confirmed and extended in several papers by Pflüger ${ }^{1}$ and others, and Pflüger laid particular stress upon the effect of the presence of sodium carbonate and of oleic acid and oleates in raising the solubilities of the other constituents.

The above experiments upon solubility of lipoid materials and their derivatives may now be considered in relationship to haemolysis.

A very considerable portion of the stroma of the red corpuscle is lipoidal in character, that is to say, is soluble in ether or similar solvents. The amount is placed at one-third of the dry weight by Pascucci, ${ }^{2}$ and of this a large amount consists of mixed lecithides, containing unsaturated fatty acids in the molecule.

Accordingly, any constituent in a serum or suspending saline which possesses the property of increasing the solubility of these lecithides must tend to lake the corpuscles by dissolving up the stroma. Such an action, as shown by Moore and Parker, is possessed by the bile salts, and they accordingly act as powerful haemolysers.

1. Arch. f. d. ges. Physiol. Bd. LXXXII, 1900, S. 303, 381; I.XXXV, 1901, S. 1 ; I.XXXVIII, 1902, S. 299, $431 ; \mathrm{XC}, 1902, \mathrm{~S} .1$.

2. Hofmeister Beiträge, 1905, Vol. VI, p. 543 ; Iscovesco ('Les Lipoides,' p. 13, 1908) places the amount of lipoids in the dried corpuscle at a lower value than one-third. 
This haemolytic power of the bile has long been known qualitatively; it has just now been followed out quantitatively in this laboratory by MacLean and Hutchinson with the most interesting results, recorded in the paper immediately succeeding this one. ${ }^{1}$

In the same fashion, we have seen that oleic acid and oleates were found experimentally to raise the solubilities of the practically insoluble palmitates and stearates in the presence of bile salts. Also, even in the absence of all bile derivatives, the solubilities in water obtained by Moore and Parker for the separate sodium salts of the acids, oleic, palmitic, and stearic on the one hand, and for the mixed sodium soaps of naturally occurring fats of pig, ox and sheep on the other, clearly show that the presence of sodium oleate increases the solubility of the other soaps.

These experimental results must be the basis of the results obtained by many observers that sodium oleate is a powerful haemolyser, while, as demonstrated by Noguchi, ${ }^{2}$ the sodium palmitate and sodium stearate are inert.

A chemical point of great importance is that both the oleic acid and the bile acids are unsaturated bodies containing in each case doubly-linked carbon atoms in an open chain, and this suggests the general law, first enunciated by St. Faust and Tallqvist, ${ }^{3}$ that the haemolytic property is associated with this absence of saturation.

At the outset of our work, we were unfortunately unaware of the existence of St. Faust and Tailqvist's paper, and we must express our regret that for th is reason we were unable in a preliminary communication ${ }^{4}$ to do justice to their most interesting work upon the subject.

These authors, in following out in a highly interesting fashion the causes of a pernicious anaemia due to the intestinal parasite, Botriocephalus latus, were able to separate from the dried bodies of the parasites a material consisting to a large extent of an unstable compound of cholesterin and oleic acid. This substance was shown to be a cholesterin ester of oleic acid of the type first separated from blood serum by Hürthle. ${ }^{5}$ This cholesterin-oleic ester had a most powerful haemolytic effect even in small quantities, and on further testing the matter, St. Faust and Tallqvist discovered that the haemolytic action was due to the oleic acid, and that sodium oleate gave a like result, while saturated soaps or their esters gave no effect upon the blood corpuscles.

1. See page 369 .

2. Noguchi, Journ. of exper. Medicine, Vol. VIII, p. 92, 1906.

3. Arch. f. exper. Path. u. Pharm., Vol. LVIJ, p. 370, 1907.

4. Journ. of Physiol,, Proc. Physiological Society, March, 1909.

5. Zeitsch. f. Physiol. Chem., Bd. XX1, 1895-6, S. 331. 
This result led the authors to the generalisation that such haemolytic action was associated with the want of saturation of the oleic acid. This was tested by employing other unsaturated acids such as acrylic, tiglic, cinnamic and erucic acids, and it was found in each case with the free acids there was marked haemolysis, although in the case of the sodium salts of tiglic and cinnamic acids there was no haemolytic activity. St. Faust and Tallqvist further demonstrated in support of their view, that when acrylic acid is hydroxylated into hydracrylic acid the latter is almost without action upon blood corpuscles.

Now one of these acids has a double bond which has been split up in the other, as shown by the formulae given below, and this is probably the cause of the difference in activity.

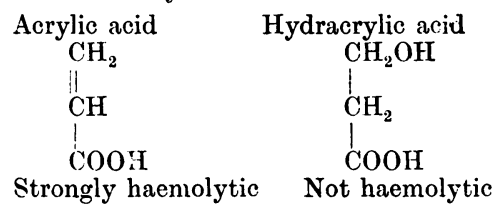

We have ourselves been able in the present series of experiments to demonstrate that an unsaturated glucoside with strongly haemolytic properties, isolated by Moore from the seeds of Bassia longifolia (Mowrah seeds), called 'Mowrin,' loses its haemolytic properties when it becomes saturated by bromination.

It is difficult to explain why the sodium salts of tiglic and cinnamic acids do not haemolyse, for they are unsaturated compounds. It may be that a certain conformation of molecule in addition to the double linkage is necessary in order to confer upon the haemolytic molecule certain physico-chemical properties which we shall subsequently see all these haemolysers possess, and that the want of saturation really confers laking power because of the physical properties of solution, etc., which attach themselves to it, and not because the double bond is broken to allow a firm combination between the two compounds.

St. Faust and Tallqvist do not appear to have tested other acids and salts than those mentioned above, nor to have proceeded to the further generalisation that a similar lack of saturation characterises other laking agents such as the bile salts and saponins.

Believing that the view is one of somewhat far-reaching importance, we have in the present experiments tested it with a number of haemolytic substances, and have always found that substances of this nature which were haemolytic were also unsaturated or possessed of a good deal of residual chemical affinity. 
Connected with the above two points of haemolytic power and want of chemical saturation, these bodies-often of widely different origin and chemical constitution-possess always a well-marked group of common properties, physical and physiological, which are so striking when placed in juxtaposition as to indicate that they and the laking process are all closely connected together and have a common cause.

Further, these common properties, which will presently be stated, are such that in spite of the fact of chemical unsaturation running through the whole group, it is difficult, or indeed impossible, to draw any definite conclusion as to whether these bodies act by forming a feeble labile chemical union, or by physically altering the properties of the solvent so that it now dissolves the lipoids.

The energy phenomenon at play is evidently one due to interaction between dissimilar chemical molecules, but whether it consists wholly, or as an initial stage, in a lowering of the surface tension at the interface between lipoid and solvent, or whether there is first a labile chemical union between lipoid and unsaturated acid causing an accumulation on the interface, so leading to a negative surface tension and hence to solution of the lipoid and to haemolysis, it is impossible to say in the present state of our knowledge.

It may be remarked, however, that the combating physical and chemical hypotheses are not so very widely apart as the two camps of adherents suppose, for in either case, the interaction is between dissimilar molecules or aggregates at an interface, and this does not differ widely from chemical action. We do not know what are the initial 'physical' stages of 'chemical' combination.

It might be asked, what is the nature of the energy change which causes accumulation of a dissolved substance on an interface and lowering of surface tension, if it be not chemical attraction and the preliminary stage of a chemical reaction? Leaving these more abstract considerations of chemical combination versus physical adsorption, we may now return to the characteristic chemical and physiological properties of the group of haemolytic agents which we are discussing.

\section{Properties of Haemolysers}

Physical Properties.-All these substances are colloidal in aqueous solution, although some of them diffuse very slowly through parchment paper; they do not crystallise out of aqueous solution, and they give rise to thick syrups as they are evaporated down to more and more concentrated 
solutions. Even in dilute solution they all froth easily, showing probably that the surface-tension is lowered. ${ }^{1}$

Chemical Properties.-All show a great tendency to form conjugated compounds, which are very easily hydrolysed by dilute acids. For example, the bile salts with amino-acids, such as glycocoll or taurin; oleic acid with cholesterin to form cholesterin esters, and with glycerol to form fats; saponin, digitalin, mowrin, and other haemolysers of that type are glucosides; the lecithides are not only conjugated compounds themselves, but unite in feeble union with a vast number of substances of biological origin, such as snake venoms and tox-albumens.

This property of conjugating chemically is, as we shall see, of the utmost importance in connection with haemolysis, where it also occurs, and may cause active haemolysis or an anti-phase, according to how it is directed.

Physiological Propertics.-The physiological properties of the whole group are closely related, and are, no doubt, dependent upon the above physical and chemical properties. Thus the soaps, the bile salts, and the whole saponin-digitalin group, are characterised by a very intensely bitter taste. Introduced directly into the circulation, they are all poisonous, and all affect the heart, causing slowing and stopping. This is in all probability due to a common cause, viz., combination with the heart lecithides.

That same physico-chemical property which attacks the red blood corpuscles by means of the attraction for its lecithin and causes haemolysis, causes attack, always of a common type with minor variations, upon the heart, due here also to chemical attraction between soap (sodium oleate), bile salt, saponin, mowrin, digitalin, or what not, of this large group of unsaturated bodies on the one hand, and the heart lecithide on the other. So variations in reactivity are caused within the heart cell, and accompanying modifications in heart beat. Here it is to be remarked that it is an integral change inside the cell of which the lecithide is a vital part that occurs, and is not a mere question of altered permeability of a lipoidal membrane.

These peculiar properties are shown in varying degree by different members of the group, but taken together they form a good set of characteristics for a very widely distributed group of substances all possessing haemolytic properties.

1. This is known to be so with the soaps, experiments with other haemolysers are in progress. 


\section{The Balancing Action of Haemolysers}

One of the most interesting experiments in haemolysis is that of Sachs and Altmann, ${ }^{1}$ demonstrating that two bodies, each of which is strongly haemolytic in itself, can be so admixed in common solution that no haemolysis whatever results, the two haemolysers balancing each other.

Thus, it was found that when sodium oleate was added in just the proper quantity to a strongly active haemolytic serum no haemolysis resulted, and that as the amount of oleate was gradually increased above this balancing amount, the mixture gradually became haemolytic again.

This result has been stated to be due to the neutralising of complement, the sodium oleate acting as an anti-complement. We think, however, that there is clear evidence against this view. In the first place, as we shall see later, an ordinary serum which is not haemolytic to the corpuscles being used, it may, in fact, be their own serum, is strongly protective against the haemolytic action of sodium oleate.

We have followed this question up in detail, as shown by the protocols of our experiments, and have successively removed or destroyed (a) immune body, (b) complement, and (c) the lipoids from the active serum. In all cases we have found that no one alone of these substances is responsible for the neutralizing of the haemolytic activity of the sodium oleate.

The further fact that not only is the laking power of the sodium oleate destroyed, but also the natural activity of the pig's serum, or the invoked activity of a specially sensitized serum, appears to us to clearly demonstrate that the soaps of the unsaturated fatty acids, oleic and linoleic, possess a selective affinity for the immune body, or actively laking, substance, of these haemolytic sera. That is to say, in the active serum the immune body and the sodium oleate or linoleate combine and mutually destroy each the other's laking power, so that the mixture in due proportion is quite inert upon the blood corpuscles.

But in case the immune bolly has been inactivated by heating, then the sodium oleate or linoleate is still captured and held by the serum proteins, so that no laking occurs until much more of the oleate or linoleate has been added than would have sufficed to cause complete and rapid laking in a saline suspension, where there is no protein to present a counter-attraction and binding agent, so that the first trace of oleate or linoleate at once attacks the lecithides and other lipoids of the corpuscles. When the serum proteins are present, although inactive themselves, they

1. Berl. klin. Wochensch., pp. 494. 699, 1908. 
form binding material for unsaturated lipoids, such as the oleates and linoleates.

The action of lecithin and cholesterin of a similar type can be explained on similar lines rather than on the view that these substances behave as active anti-complements.

On the other hand, as our experiments also prove, two lipoids of nearly allied nature which do not therefore combine with each other, or mutually adsorb each other, such as oleate and linoleate of sodium, show no balancing action whatever, but produce a distinctly additive effect. So that whether shown by the smaller amounts which will produce complete laking in a given time in presence of each other, or better by observing the laking times of two minimal amounts of oleate and linoleate separately, as compared with the time spent for laking with the halves of these amounts acting in consort, the result always comes out that the haemolytic effect consists of the two added factors of oleate action and linoleate action; there being no reduction whatever due to action between the two haemolysers, such as is seen between either of them and the active haemolytic body of a.sensitized serum or a serum naturally haemolytic.

We may hence enunciate the law that if two given haemolysers are capable of combining or adsorbing with each other, they will tend to balance each other, and the effect on corpuscles will be less than either acting alone; but if no adsorption is possible between the two, the effect in common solution upon the corpuscles will be the sum of the effects of the two.

\section{EFfects of Oxidizing and) Redecing Agents upon ILaemolysts}

These experiments were suggested by analogies between the mode of action of the peroxidases and haemolytic serum, in that heating to $56^{\circ}$ C. destroys the tissue peroxides and so stops the action of the peroxidases in a somewhat similar way to that in which heating to $56^{\circ} \mathrm{C}$. inactivates a haemolytic serum by destroying complement.

The results of experiment showed that it was not possible to replace the destroyed complement of an inactivated serum by means of hydrogen peroxide or other form of peroxide, so that the haemolytic agent or immune body can hardly be regarded as a peroxidase ferment.

Yet the experiments yielded the very interesting information that addition of an alkaline reducing agent, such as ammonium sulphide, even in very small amount, entirely inactivated an active serum, and contrariwise an oxidizing agent, such as hydrogen peroxide in alkaline 
solution, very much increased the haemolytic power. The peroxide alone, or hydrogen sulphide alone, in absence of alkali, had very little action; but the addition of a trace of ammonia at once produced the inhibiting action in the case of the sulphide, or favouring action in the case of the peroxide.

In view of the fact that ammonia and other alkalies by themselves possess a laking effect, it may be emphasized that the amounts being used lay below the laking amounts when used alone, as shown by control experiments.

\section{Experimentai Methods and Results}

The sodium salts of five fatty acids were taken, namely, the sodium salts of stearic, palmitic, erucic, oleic and linoleic acids.

In this list the first two are sodium soaps of saturated fatty acids, belonging to the acetic acid series; sodium erucate and oleate belonging to the acrylic series, are unsaturated sodium soaps, each having one doublylinked carbon atom in their formula; sodium linoleate belonging to the linolic acid series is still more unsaturated, having three doubly-linked carbon atoms in its constitution.

To test the haemolytic power of these soaps, solutions varying in strength from $0.01 \mathrm{M}$ to $0.001 \mathrm{M}$ were made up by the simple procedure of weighing out the requisite amount of pure free acid and neutralising with the calculated amount of decinormal alkali. Thus, taking $\mathrm{C}_{18} \mathrm{H}_{34} \mathrm{O}_{2}$ as the formula for oleic acid, this gives a molecular weight of 282 , which is equivalent to 0282 grams in 100 c.c. for a centimolecular solution. This weight of oleic acid was therefore weighed out in a beaker and neutralised with.10 c.c. of $0.1 \mathrm{M} \mathrm{Na(OH}$, and the volume made up to 100 c.c. by adding normal isotonic saline solution. The weaker molecular strengths were made up by adding proportionally more normal saline. The other sodium soaps were made up in a similar manner.

For the experiment a series of test-tubes were taken, and in each was placed a certain known quantity of the sodium soap, whose haemolytic properties it was desired to test, and 1 c.c. of a 5 per cent. emulsion of sheep's red blood corpuscles; the volume of each tube was then made up to 5.5 c.c. by adding normal isotonic saline solution. When the contents of the tube were completed, they were placed in a thermostat at a temperature of $37^{\circ} \mathrm{C}$. and observations made.

It should be mentioned that in this and the subsequent experiments the emulsion of sheep's red blood corpuscles was made by defibrinating 
fresh blood and then washing and centrifuging the corpuscles three times in normal saline, and finally the washed red blood corpuscles were made up into a 5 per cent. emulsion in normal isotonic saline.

The results of several experiments with the soaps above mentioned gave the following results:--

In the tubes containing:
0.2 c.c. $\frac{M}{100}$ sodium stearate. No haemolysis in 24 hours.
0.2 c.c. $\frac{\mathrm{M}}{100}$ sodium palmitate. No haemolysis in 24 hours.
$0 \cdot 2$ c.c. $\frac{\mathrm{M}}{100}$ sodium erucate. Complete haemolysis in 24 hours.
0.8 c.c. $\frac{M}{1000}$ sodium erucate. No haemolysis in 24 hours.
0.2 c.c. $\frac{\mathrm{M}}{100}$ sodium oleate. Complete haemolysis in 1 hour.
0.4 c.c. $\frac{M}{1000}$ sodium oleate. Complete haemolysis in 3 hours.
0.2 c.c. $\frac{M}{1000}$ sodium oleate. Slight amount of laking after 20 hours.
0.4 c.c. $\frac{M}{4000}$ sodium oleate. Merest trace of laking after 20 hours.
0.2 c.c. $\frac{M}{100}$ sodium linoleate. Complete haemolysis in 16 minutes.
0.8 c.c. $\frac{\mathrm{M}}{1000}$ sodium linoleate. Complete haemolysis in 50 minutes.
0.4 c.c. $\frac{\mathrm{M}}{1000}$ sodium linoleate. Complete haemolysis in 153 minutes.
0.2 c.c. $\frac{M}{1000}$ sodium linoleate. Complete haemolysis in 20 hours.
0.4 c.c. $\frac{M}{4000}$ sodium linoleate. Slight amonnt of laking in.24 hours

It will be seen that in the case of the saturated sodium soaps of stearic and palmitic acid no haemolysis was observed with the above strengths, but with very much weaker strengths of the unsaturated soaps complete haemolysis was obtained, and that sodium linoleate, which has the greater number of doubly-linked carbon atoms, possesses also the strongest laking action, completely haemolysing the sheep's red blood corpuscles within twenty hours, even in a concentration equivalent to $0 \cdot 000004 \mathrm{M}$. Sodium oleate, which has one doubly-linked carbon atom, is also a powerful haemolytic agent, but not so active as sodium linoleate, though more powerful than sodium erucate, which is an equally unsaturated soap, but with a different molecular constitution, and with corresponding physical 
properties showing less typically the common character of the class of haemolysers.

On the same grounds, some observations were also made with a glucoside mowrin and the sodium salt of mowric acid, one of the products prepared from the glucoside by hydrolysis. These preparations, which are unsaturated bodies, were prepared by Moore from the seeds of Bassia longifolia, commonly known as Mowrah seeds.

The following are the haemolytic results obtained with these substances, using 1 c.c. of 5 per cent. emulsion of sheep's red blood corpuscles and a total volume of 5.5 c.c., and following the same technique as in the previous experiments :-
1 c.c. $\frac{M}{100}$ Mowrin.
Complete haemolysis within 1 minute.
0.75 c.c. $\frac{\mathrm{M}}{10000} \quad$,
Complete haemolysis within 4 hours.
0.5 c.c. $\frac{M}{10000} \quad$,
No haemolysis in 24 hours.
1 c.c. $\frac{\mathrm{M}}{100}$ Sodium Mowrate.
Complete haemolysis in 15 minutes.
1 c.c. $\frac{\mathrm{M}}{1000}$
Trace of haemolysis in 20 hours.
0.75 c.c. $\frac{M}{1000} \quad, \quad, \quad$ No haemolysis.

It will thus be seen again that these unsaturated bodies, especially the glucoside mowrin, are also powerful haemolytic bodies.

If, however, the sodium salt of mowric acid is brominated, the haemolytic action is markedly weakened, for instance, 1 c.c. of $0.01 \mathrm{M}$ sodium mowrate haemolyses in fifteen minutes, but of an exactly similar quantity if the brominated sodium mowrate be used, the time required in this case for complete haemolysis is five hours.

In view of the fact that there are present in the normal organism many unsaturated haemolytic lipoids, it is of interest to note the protective action that the animal's serum is able to exert on behalf of its own red blood corpuscles.

In order to study this action as regards the three unsaturated haemolytic soaps used in the previous experiments, a series of test-tubes were taken in which various haemolytic quantities of these soaps were placed, and 3 c.c. of fresh sheep's serum added to each tube; the serum and soap were then incubated together for half an hour at $57^{\circ} \mathrm{C}$., after which the sheep's corpuscles were added and the tubes replaced in the thermostat; no haemolysis occurred in any of the tubes, even though 
1 c.c. $0 \cdot 01 \mathrm{M}$ of each soap was used, which amount alone would in the case of sodium oleate and linoleate have laked an equal quantity of sheep's red blood corpuscles almost instantaneously, and eight minutes would have sufficed for sodium erucate.

Further observations showed that 05 c.c. sheep's serum will exactly protect 1 c.c. 5 per cent. emulsion of sheep's red blood corpuscles against 0.35 c.c. of $0.01 \mathrm{M}$ sodium linoleate. Sheep's serum will also protect its own corpuscles against the natural haemolytic action of pig's serum; for example, we found that 1 c.c. of 5 per cent. emulsion of sheep's red blood corpuscles is completely haemolysed by 0.5 c.c. fresh pig's serum within an hour, the addition, however, of 2 c.c. of sheep's serum will completely inhibit this action.

Chclesterin also has an anti-haemolytic action, though not very marked, 1 c.c. $0.002 \mathrm{M}$ cholesterin emulsion being able to inhibit the action of 0.7 c.c. $0 \cdot 001 \mathrm{M}$ sodium oleate. Difficulty was experienced in obtaining the cholesterin in a suitable medium to work with, as the solvents of this compound, such as acetone, etc., are mostly haemolytic. In these experiments, therefore, an emulsion of finely suspended cholesterin in normal saline was used, its strength being approximately $0.01 \mathrm{M}$.

This protective action of serum and cholesterin will be again referred t. later on, when we shall have pointed out an action which Sachs and Altmann first described in the case of sodium oleate, and which they termed the behaviour of sodium oleate as anti-complement.

Experiments are described below showing not only that this action can be extended to other unsaturated soaps, but also that it is, to a certain extent, independent of the presence of either complement or amboceptor, and therefore the term 'anti-complement' has been omitted and the word 'balancing' used in its place, as more accurately describing the action. For not only is the haemolytic property of the pig's serum on sheep's red blood corpuscles gradualiy inhibited as the amount of soap increases, but also after this action has been completely balanced, the serum on its own part further inhibits the haemolytic action of the soap.

Pig's serum is naturally haemolytic for sheep's red blood corpuscles, but if to pig's serum is added a certain quantity of sodium oleate there is an inhibition of haemolysis, and a point can be found where, owing to interaction between these two substances, no haemolysis occurs, although the quantities used of each haemolytic agent are such that if either was use: separately complete laking of the sheep's red blood corpuscles would ensue. 
For instance, tubes containing:-

a. 1 c.c. sheep's red blood corpuscles-0.5 c.c. pig's serum -4 c.c. normal saline gives complete haemolysis within half an hour.

b. 1 c.c. sheep's red blood corpuscles -0.7 c.c. $\frac{M}{100}$ sodium oleate-3.8 c.c. normal saline results in complete haemolysis in 3 minutes.

But :-

c. 1 c.c. sheep's red blood corpuscles -0.7 c.c. $\frac{M}{100}$ sodium oleate-0.5 c.c. pig's serum -3.3 c.c. normal saline results in almost complete inhibition of haemolysis.

The same holds good for sodium linoleate, and sodium erucate, though the quantities vary in each case.

The results of an experiment are shown graphically in fig. 1. Many similar experiments were carried out giving parallel results :-

In all cases the sodium salt of the acid, the serum and saline were incubated together for three-quarters of an hour at $37^{\circ} \mathrm{C}$. prior to adding the sheep's red blood corpuscles. When the contents of the tubes were completed they were again placed in the thermostat at $3 \tau^{\circ} \mathrm{C}$, and observations made from time to time.

The slight variations in these results are probably due to variations in the 'titer' of the different supplies of pig's serum that were used, as the solutions of oleate and linoleate of soda were the same in each experiment, and, moreover, it may be mentioned that the difference between the balancing points for the two unsaturated soaps is constant in all experiments, in exach the amount of $0.01 \mathrm{M}$ sodium linoleate required to balance 0.5 c.c. pig's serum being 0.3 c.c. in excess of the quantity of sodium oleate required for the same result.

It is interesting to note that although according to the previous experiments sodium linoleate alone is a stronger haemolytic agent than sodium oleate, yet it does not seem to be as powerful as the oleate in balancing the action of pig's serum.

These experiments, while showing the balancing action of sodium oleate and linoleate, seemed also to point to the existence of another inhibitory action which might be independent of the existence of complement in the pig's serum.

Some pig's serum was therefore inactivated by heating it at $56^{\circ} \mathrm{C}$. for half an hour, and after making sure that the serum was completely inactivated, exactly similar experiments were carried out as before. It was then found that the haemolytic action of the sodium liroleate was inhibited up to the same point when using the inactivated as 


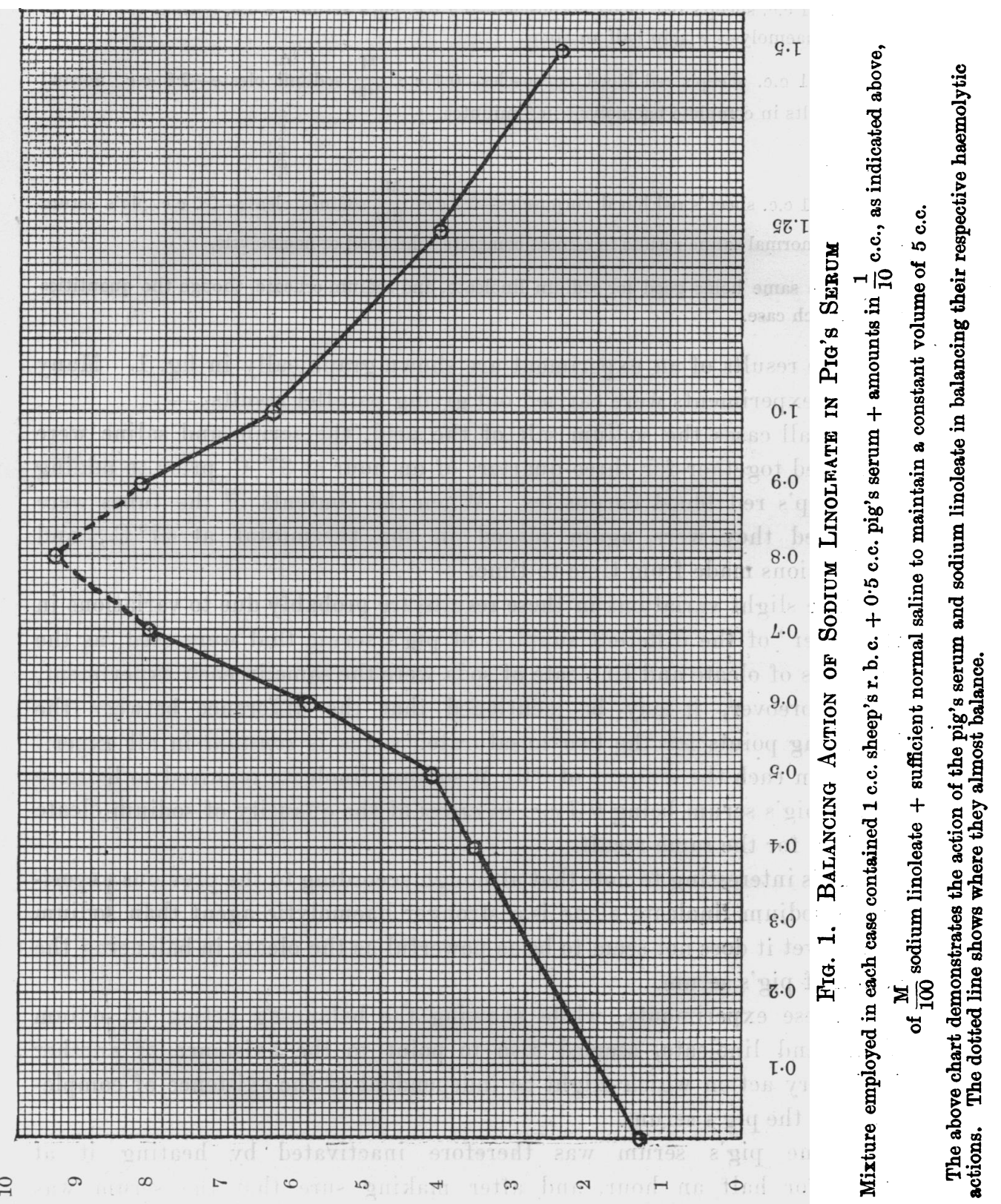




when using the fresh serum; that is to say, whereas 1 c.c. $0 \cdot 01 \mathrm{M}$ sodium linoleate will alone haemolyse 1 c.c. of 5 per cent. emulsion of sheep's red blcod corpuscles almost instantaneously, yet when used with either 0.5 c.c. of the fresh or the inactivated pig's serum its action was almost completely inhibited. As a further step, some pig's serum was taken in which both the complement and the amboceptor had been removed. This was accomplished by heating some pig's serum to $55^{\circ} \mathrm{C}$. for half an hour to destroy the complement, and then adding excess of sheep's red blood corpuscles (fresh pig's serum being powerfully haemolytic for sheep's red blood corpuscles), incubating the mixture together for an hour at $37^{\circ} \mathrm{C}$. and then centrifuging; the clear supernatant serum being again treated with sheep's red blood corpuscles until by tests it was evident that all the amboceptor had combined with the corpuscles, and the clear serum contained neither of its two haemolytic factors.

The effect of this serum on the haemolytic properties of sodium lincleate was then tested in an exactly similar way to that used in the immediately preceding experiments, and it was found that even thus depleted it maintained its protective power intact, and, moreover, that this factor was not in any way diminished. With these results before us, it will be at once apparent that although in the fresh state pig's serum is a powerful haemolyser of sheep's corpuscles, yet if we remove from this serum one or both of its haemolytic factors it then exerts a powerful protective action on the red blood corpuscles, an action which in the case of the sheep's red blood corpuscles is more potent than that exercised by the animal's own serum, for it will be observed that 0.5 c.c. of sheep's serum was able to protect 1 c.c. of a 5 per cent. emulsion of sheep's red blood corpuscles against the haemolytic action of 0.35 c.c. $0.01 \mathrm{M}$ sodium lir:cleate, while under similar conditions 0.5 c.c. of inactivated pig's serum was able to completely inhibit the haemolytic action of 0.6 c.c. $0.01 \mathrm{M}$ sodium linoleate on a similar quantity of sheep's red blood corpuscles.

We have already mentioned that cholesterin has the power of inhibiting, up to a certain point, the haemolytic action of various soaps.

The question arises, is the above protective action due, as Iscovesco thinks, to cholesterin?

An attempt to investigate this by trying to extract this inhibitory body from pig's serum by means of ether gave a negative reply to this question.

To carry out this experiment 100 c.c. of fresh pig's serum was poured into a separating funnel and 150 c.c. of ether added, the contents being then shaken up together for fifteen minutes, allowed to stand, and then the 
serum which collected in the lower part of the funnel was withdrawn. This process was repeated three times, using fresh ether each time. The three portions of ether were then collected into one flask and the ether slowly distilled off at a temperature of $37^{\circ} \mathrm{C}$, the last traces being removed by means of a suction pump. The residue containing ether extractives was then shaken up with 30 c.c. of warm normal saline, forming thereby a white opalescent soapy emulsion.

The action of this emulsion was tried on some sheep's red blood corpuscles, but it was found neither to have any haemolytic action alone nor any inhibitory action against other laking agents.

The serum, which had been carefully separated from the ether, was then placed in a flask and all trace of ether removed by bubbling air throug?, it was then tested with sheep's red blood corpuscles, and it was found that although now it had no haemolytic power, yet its protective action was intact.

The result would appear to be evidence indicating that although cholesterin undoubtedly has considerable inhibitory power, yet it does not account for the protective action of the serum, as the extraction with ether would probably have removed the greater portion.

\section{Additive Effect on Haemolysis of Two Closely Aluied Haemolysers which cannot therefore Combine with Each Other}

The preceding experiments show that two dissimilar haemolysers, such as the haemolytic substance of pig's serum for sheep's corpuscles and sodium oleate or linoleate, so far from supplementing, balance each other.

The present experiment demonstrates that sodium oleate and sodium linoleate uscd in common solution aid each other, the effect being approximately additive.

Thus, using the same technique as previously described:-

I. Sodium oleate, 1.25 c.c. of $0.001 \mathrm{M}+$ sheep's r. b. c. 1 c.c. of 5 per cent. emulsion + normal saline to 5 c.c. Result-complete laking in 26 minutes.

II. Sodium linoleate, 1 c.c. of $0.001 \mathrm{MI}+$ sheep's r. b. c. 1 c.c. of 5 per cent. emulsion + normal saline to 5 c.c. Result-complete laking in 19 minutes.

III. The same quantities of the two together, viz., sodium oleate 1.25 c.c. of $0.001 \mathrm{MI}$ + sodium linoleate 1 c.c. of $0.001 \mathrm{M}+$ sheeps r. b. c. 1 c.c. of 5 per cent. emulsion + saline to 5 c.c. Result-complete laking in 11 minutes.

$I V$. Half the initial amounts of the two sodium salts gave the following results, viz. :Sodium oleate, 0.62 c.c. of $0.001 \mathrm{M}+$ sodium linoleate 0.5 c.c. of $0.001 \mathrm{M}+$ sheep's r. b. c. 1 c.c. of 5 per cent. emulsion + saline to 5 c.c. Result-laking in 28 minutes.

There is accordingly no balancing here, and the effects of the two used in common solution are practically a purely additive function. 
When sodium linoleate and fresh lecithin are used as a pair of haemolysers, a very distinct balancing action is obtained. Thus, $0 \cdot 2$ c.c. of $0.01 \mathrm{M}$ sodium linoleate laked 1 c.c. of 5 per cent. emulsion of sheep's red blood corpuscles in four minutes; but when exactly the same amount of the sodium linoleate is first treated with 0.5 c.c. of an emulsion corresponding to $0.01 \mathrm{M}$ lecithin, the haemolysis is delayed for about two and three-quarter hours.

This illustrates very clearly the constituent in the red blood corpuscle which is attacked in haemolysis, and also shows that the haemolyser and the lecithin of the corpuscles enter into at least a quasi-combination, so limiting the amount of corpuscle haemolysable by a given amount of haemolyser.

\section{Relationships of Oxidizing and Reducing Agents to Haemolysis}

Hydrogen Peroxide and Haemolysis.-At the outset of this investigation an attempt was made to test whether complement could be replaced by hydrogen peroxide.

Pig's serum, which, as is well known, has a haemolytic action on sheep's red blood corpuscles, was inactivated, and to different dilutions of serum varying strengths of hydrogen peroxide were added. Repeated experiments yielded discordant results; a strength of hydrogen peroxide which on one occasion caused haemolysis, failed to do so a second time.

Investigation showed that the hydrogen peroxide had an acid reaction, and this was probably the cause of the uncertain results.

On using dilutions of Merck's perhydrol, much more uniform results were obtained. Except in very strong solutions--up to 1 in 50 in normal saline-perhydrol had no haemolytic action on fresh sheep's red corpuscles. Added to inactivated pig's serum it had no complementary action, and, indeed, the haemolytic action of the stronger solutions seemed to be inhibited by the serum.

Another oxidizing agent, quinone, was tried in varying strengths in a similar way. No haemolytic or complementary action was obtained.

Added to fresh pig's serum, neither perhydrol nor quinone interfered with its haemolytic power.

Similarly, oxidase containing solutions from fresh vegetable juices could not be used as substitutes for complement.

Thus, we have been unable to trace the nature and action of complement in haemolysis, but our experiments led us incidentally to certain observations upon the effects of reducing and oxidizing substances on haemolysis which are here recorded. 


\section{Effect of Reducing Agents on Complement}

We next tried the effect of various dilutions of ammonium sulphide on the haemolytic action of pig's and other sera. The stock ammonium sulphide used for dilutions was of a strength $0.4 \mathrm{M}$. Table I gives the details of the experiments. The results show that in dilution up to 1 in 1,000 the ammonium sulphide inhibits the haemolytic action of the sera used.

Equivalent solutions of hydrogen sulphide and ammonia in normal saline have not such inhibitory action.

In all experiments the tubes were all made up to 5 c.c. with normal saline.

The sheep's red corpuscles were used in the form of a 5 per cent. suspension in normal saline.

Fresh guinea-pig's serum was used as complement.

The anti-sheep haemolytic rabbit's serum was of such a titre that 1 c.c. of a 1 in 1,500 dilution dissolved 1 c.c. of a 5 per cent. suspension of sheep's red blood corpuscles in half an hour.

\section{TABIF I}

1. 1 c.c. of fresh pig's serum +1 c.e. ammonium sulphide (dilution 1 in 50$)+1$ c.c. r. b. c. - No hremolyzis

2.

3.

4. (dilution 1 in 100)

(dilution 1 in 200)

(dilution 1 in 300 )



,

, Partial haemolysis

5. 1 c.c. of inactivated serum ! +1 c.c. ammonium sulphide (dilution 1 in 50 ) +1 c.c. r. b. c. - No haemolysis $+0 \cdot 1$ c.c. complement

6.

7.

8.

,

,

" (dilution 1 in 100)

(dilution 1 in 200)

(dilution 1 in 300)

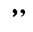

,

,
Haemolysis "

9. 1 c.c. inactivated rabbit's serum (dilution 1 in 20 ) ! +1 c.c. ammonium sulphide +1 c.o. r. b. c. - No haomolysis $+0 \cdot 1$ c.c. complement (dilution 1 in 50 )

10.

11.

12.

”,
(dilution 1 in 100) (dilution 1 in 200) (dilution 1 in 300)

"
,"

Slight haemolysis Complete haemolysis

13. 1 c.c. fresh pig's serum +1 c.c. $r$. b. c.

14. 1 c.c. inactivated pig's serum $+0 \cdot 1$ c.c. complement +1 c.c. r. b. c. $\quad \ldots$

15. 1 c.c. inactivated rabbit's serum $+0 \cdot 1$ c.c. complement +1 c.e. r. b. c. $\quad \ldots \quad \ldots$

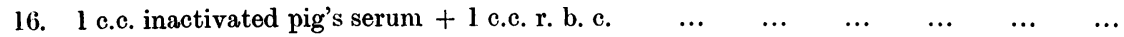

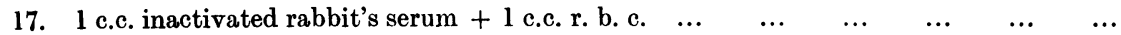

18. 1 c.c. ammonium sulphide (dilution 1 in 50 ) +1 c.c. r. b. c.

All tubes were made up to 5 c.c. with normal saline.

The sera and ammonium sulphide were incubated together at $37^{\circ} \mathrm{C}$. for half an hour, r.b.c. emulsion then added, and whole incubated for one hour. 
A comparison of the effects of equivalent strengths of sodium hydrooxide, ammonium hydrate, and ammonium sulphide on sheep's r.b.c. are given by Table II..
TABLE II
1. 0.5 c.c. $\frac{\mathrm{N}}{10} \mathrm{NaOH}+1$ c.c. r. b. c. - Haemolysis in half an hour
2. 0.5 c.c. $\frac{\mathrm{N}}{10} \mathrm{NH}_{4} \mathrm{OH}+1$ c.c. r. b. c. - Slight haemolysis next day
3. 0.5 c.c. $\frac{\mathrm{N}}{10}\left(\mathrm{NH}_{4}\right)_{2} \mathrm{~S}+1$ c.c. r. b. c. - No haemolysis

All tubes made up to 5 c.c. with normal saline.

In Tables III, IV and V are shown the 'balancing' action of sodium hydrate with fresh and inactivated serum; also the influence of perhydrol on this action. All tubes were made up to 5 c.c. with normal saline, incubated at $37^{\circ} \mathrm{C}$. for two hours, and results noted.

\section{TABLE III}

1. 1 c.c. fresh pig's serum +1 c.c. r. b. c.

2.

$\begin{array}{ll}\cdots & \cdots \\ +0.5 \text { c.c. } & \frac{\mathrm{N}}{10} \mathrm{NaOH}\end{array}$

$$
+0.5 \text { c.c. } \frac{\mathrm{N}}{15} \mathrm{NaOH}
$$

3.

4.

$$
\begin{aligned}
& +0.5 \text { c.c. } \frac{\mathrm{N}}{20} \mathrm{NaOH} \\
& +0.5 \text { c.c. } \frac{\mathrm{N}}{25} \mathrm{NaOH}
\end{aligned}
$$

5.

6.

7. 0.5 c.c. $\frac{\mathrm{N}}{10} \mathrm{NaOH}+1$ c.c. r. b. c. ...

+0.5 c.c. $\frac{\mathrm{N}}{30} \mathrm{NaOH}$

8. 0.5 c.c. $\frac{\mathrm{N}}{15} \mathrm{NaOH}+1$ c.c. r. b. c. ...

9. 0.5 c.c. $\frac{\mathrm{N}}{20} \mathrm{NaOH}+1$ c.c. r. b. c. ...

10. 0.5 c.c. $\frac{\mathrm{N}}{25} \mathrm{NaOH}+1$ c.c. r. b.

11. $0 \cdot 5$ c.c. $\frac{\mathrm{N}}{30} \mathrm{NaOH}+1$ c.c. r. b. c. ...

\section{TABLE IV}

l. 1 c.c. fresh pig's serum +1 c.c. $r$ b. c.

2. 0.5 c.c. $\frac{\mathrm{N}}{10} \mathrm{NaOH}+1$ c.c. r. b. c.

3. 1 c.c. fresh pig's serum +0.5 c.c. $\frac{\mathrm{N}}{10} \mathrm{NaOH}+1$ c.c. r. b. c.

4.

5.

\section{:}

?

7.

,

"
,
+1 c.c. perhydrol ( 1 in 30 dilution)

+1 c.c. perhydrol

( 1 in 60 dilution)
Haemolysis

No haemolysis

Haemolysis

,

"

"

,

,

No haemolysis

, 


\section{Table V}

1. 1 c.c. inactivated pig's serum +0.5 c.c. $\frac{\mathrm{N}}{10} \mathrm{NaOH}+1$ c.c. r. b. c.

Slight haemolysis in 4 hours

2.

3

4.

5 .

$$
\begin{aligned}
& \text { " } \\
& \text {, }, \\
& +1 \text { c.c. r. b. c. }+1 \text { c.c. perhydrol } \\
& \text { ( } 1 \text { in } 30 \text { dilution) } \\
& +1 \text { c.c. perhydrol } \\
& \text { ( } 1 \text { in } 60 \text { dilution) }
\end{aligned}
$$

+1 c.c. perhydrol Haemolysis in 2 hours ( 1 in 30 dilution)

+1 c.c. perhydrol Haemolysis in 2 hours ( 1 in 60 dilution)

The haemolytic action of ammonia in various strengths on sheep's r.b.c. is shown in Table VI.

\section{Table VI}

1. 3 c.c. $\frac{\mathrm{N}}{10}$ ammonia +1 c.c. r. b. c. ... ... Haemolysis in 2 or 3 minutes

2. 2 c.c.

3. 1.5 c.c. "

4. 1 c.c. ", ,

5. 0.75 c.c. , ,

6. 0.5 c.c.

All tubes were made up to 5 c.c. with normal saline.

Table VII gives the effects of adding ammonia to fresh and inactivated pig's serum, and of variations in the technique.

\section{TABLe VII}

1. 1 c.c. $\frac{\mathrm{N}}{10}$ ammonia +1 c.c. fresh pig's serum $\ldots \quad \ldots \quad \ldots$ Slight haemolysis next day

2. 0.75 c.c.

3. 0.5 c.c.

4. I c.c. $\frac{\mathrm{N}}{10}$ ammonia +1 c.c. inactivated pig's serum ... Very slight haemolysis next day

5. 0.75 c.c.

6. 0.5 c.c.

Tubes made up to 4 c.c. with normal saline, incubated for one hour at $37^{\circ} \mathrm{C}$, then 1 c.c. r.b.c. added to each tube and incubated for two hours.

7. 1 c.c. $\frac{\mathrm{N}}{10}$ ammonia +1 c.c. fresh pig's serum +1 c.c. r. b. c. Slight haemolysis next day

8. 0.75 c.c.

9. 0.5 c.c.

... Very slight haemolysis next day

10. 1 c.c. $\frac{N}{10}$ ammonia +1 c.c. inactivated pig's serum +1 c.c. r. b. c. Pale brown colour

11. $0 \cdot 75$ c.c.

12. 0.5 c.c.

Tubes made up to 5 c.c. with normal saline and r.b.c. at once, and incubated for two hours. 
Having found that quinone alone did not much influence haemolysis, we next tried the effect of quinone with ammonia added. The alkali promptly turns the quinone black, or brown in weak dilutions, probably turning it into hydroquinone, which then undergoes some further change. Quinone added to a dilution of ammonia, which by itself had no haemolytic action in two hours, promptly turned black and produced instant haemolysis. Inactivated pig's serum inhibits this action to some extent. Hydroquinone alone has no haemolytic effect, but with ammonia produces the same results as quinone. The results obtained are set forth in Table VIII.

\section{TaBLE VIII}

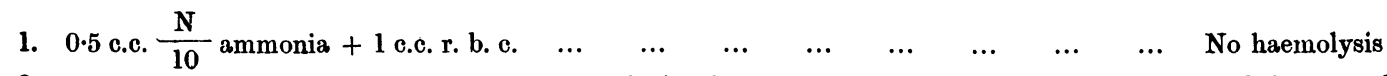

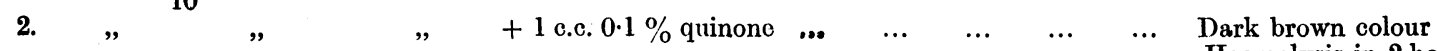

Haemolysis in 2 hours

3. 0.2 c.c.

4. $0 \cdot 1$ c.c.

5. 0.5 c.c. ,

6. 1 c.c. $0 \cdot 1 \%$ quinone +1 c.c. r. b. c.

7. 1 c.c. $0.1 \%$ hydroquinone +1 c.c. r. b. c.

8

9. 0.5 c.c. $\frac{\mathrm{N}}{10}$ ammonia +1 c.c. r. b. c. +1 c.c. $0.1 \%$ hydroquinone $\quad \ldots \quad \ldots \quad \ldots \quad \begin{gathered}\text { Black colour } \\ \text { Haemolysis }\end{gathered}$

10.

1 $=\quad, \quad, \quad+1$ c.c. inactivated pig's serum

Brown colour No haemolysis

1 0.1 c.c. $\frac{\mathrm{N}}{10}$ ammonia +1 c.c. r. b. c. +1 c.c. $0 \cdot 1 \%$ hydroquinone ... Dark brown colour No haemolysis

12. $"$ " $\quad$ " $\quad$, + 1 c.c. inactivated pig's serum $\begin{aligned} & \text { Brown colour } \\ & \text { No hremolysis }\end{aligned}$ All tubes made up to 5 c.c. with normal saline.

\section{Stimmary of Results}

1. The substances concerned in haemolysis, including thereby both the haemolytic agent outside and the substance attacked within, have a powerful mutual effect upon one another's solubilities.

2. Instances are given of such increased solubilities, and the favouring effects upon haemolysis noted. As a result of such increased solubility lecithides are dissolved out from the mass of the corpuscle, so setting free the haemoglobin also, and laking is the result.

3. It is noted that all the haemolytic class of unsaturated soaps of 
fatty acids, saponin, mowrin, digitalin, the various bile salts, possess common physical, chemical and physiological properties, and are all unsaturated bodies capable of bromination, etc.

4. The similar action upon the heart of the haemolytic bodies is probably due to combination between these and the heart lipoids.

5. Although want of saturation exists, it is probable that the first fundamental step is a 'physical' one of lowering of surface tension with accompanying tendency to solution. But no hard and fast line can be drawn between so-called physical and chemical action.

6. The balancing action of haemolysers is discussed, and it is shown that this is not obtained with closely similar haemolysers, where instead an additive action is seen. This suggests that balancing is due to a combination or interlocking of the two haemolysers, whereby nothing is left free to touch the corpuscles.

7. Where sera and such haemolysers as sodium oleate balance, the first call is between the active body of the serum and the oleate; next, in absence of the active body or of complement, the serum proteins alone, although not active in themselves as haemolysers, possess a superior binding power over the corpuscles for the oleate, and hence act as protectors, so that much more oleate in excess must be added before the corpuscles are attacked. Accordingly, as is well known, a mere trace of oleate suffices to break down corpuscles in saline suspension, but in serum suspension many times more oleate must be added before any result is obtained.

8. It follows from this that oleates, etc., do not act as anti-complements, and ought not to be described as such; it is most probable that they possess no specific relationship whatever to complement.

9. Sodium oleate can also be balanced by lecithin for similar reasons.

10. Under conditions specified, and in alkaline solution, oxidizing agents favour haemolysis, and reducing agents restrain it; but an oxidizing agent alone cannot replace complement in an active haemolytic serum, and it is not probable that complement has the nature of a peroxide body. 